STRUCTURAL BIOLOGY COMMUNICATIONS

ISSN 2053-230X

Received 1 November 2017

Accepted 16 February 2018

Edited by M. J. Romao, Universidade Nova de Lisboa, Portugal

Keywords: ancestral sequence reconstruction; pyruvate decarboxylase; lyases; crystal structure; TPP-dependent enzymes.

PDB reference: inferred ancestral pyruvate decarboxylase, $5 \mathrm{npu}$

Supporting information: this article has supporting information at journals.iucr.org/f

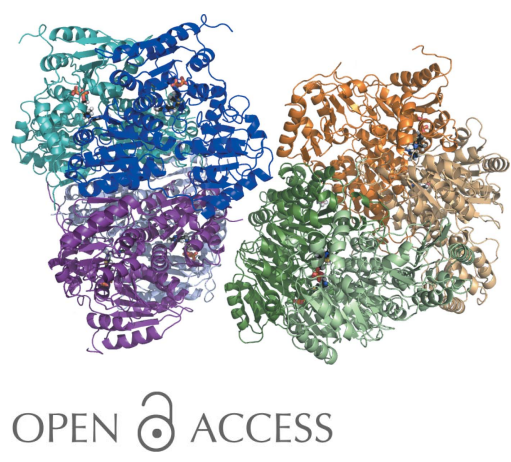

\section{Crystal structure of an inferred ancestral bacterial pyruvate decarboxylase}

\author{
Lisa Buddrus, ${ }^{\text {a,b }}$ Emma S. V. Andrews, ${ }^{\mathrm{c}}$ David J. Leak, ${ }^{\mathrm{b}}$ Michael J. Danson, \\ Vickery L. Arcus ${ }^{\mathrm{c}}$ and Susan J. Crennell ${ }^{\mathrm{b} *}$

\begin{abstract}
${ }^{\mathbf{a}}$ School of Biochemistry, University of Bristol, University Walk, Bristol BS8 1TD, England, ${ }^{\mathbf{b}}$ Department of Biology and Biochemistry, University of Bath, Claverton Down, Bath BA2 7AY, England, and ${ }^{\mathbf{c}}$ School of Science, Faculty of Science and Engineering, University of Waikato, Private Bag 3105, Hamilton 3240, New Zealand. *Correspondence e-mail:
\end{abstract} \\ s.j.crennell@bath.ac.uk
}

Pyruvate decarboxylase (PDC; EC 4.1.1.1) is a key enzyme in homofermentative metabolism where ethanol is the major product. PDCs are thiamine pyrophosphate- and $\mathrm{Mg}^{2+}$ ion-dependent enzymes that catalyse the non-oxidative decarboxylation of pyruvate to acetaldehyde and carbon dioxide. As this enzyme class is rare in bacteria, current knowledge of bacterial PDCs is extremely limited. One approach to further the understanding of bacterial PDCs is to exploit the diversity provided by evolution. Ancestral sequence reconstruction (ASR) is a method of computational molecular evolution to infer extinct ancestral protein sequences, which can then be synthesized and experimentally characterized. Through ASR a novel PDC was generated, designated ANC27, that shares only $78 \%$ amino-acid sequence identity with its closest extant homologue (Komagataeibacter medellinensis PDC, GenBank accession No. WP_014105323.1), yet is fully functional. Crystals of this PDC diffracted to $3.5 \AA$ resolution. The data were merged in space group $P 3_{2} 21$, with unit-cell parameters $a=b=108.33, c=322.65 \AA$, and contained two dimers (two tetramer halves) in the asymmetric unit. The structure was solved by molecular replacement using PDB entry $2 \mathrm{wvg}$ as a model, and the final $R$ values were $R_{\text {work }}=0.246$ (0.3671 in the highest resolution bin) and $R_{\text {free }}=0.319(0.4482$ in the highest resolution bin). Comparison with extant bacterial PDCs supports the previously observed correlation between decreased tetramer interface area (and number of interactions) and decreased thermostability.

\section{Introduction}

Pyruvate decarboxylase (PDC; EC 4.1.1.1) is a thiamine pyrophosphate- and $\mathrm{Mg}^{2+}$ ion-dependent enzyme that catalyses the non-oxidative decarboxylation of pyruvate to acetaldehyde and carbon dioxide. Bacterial PDCs are key enzymes in homofermentative metabolism where ethanol is the main fermentation product, and as such are of great interest for biotechnological applications, including second-generation bioethanol production for use as a renewable transport fuel. In recent years there has been growing interest in utilizing bacterial PDCs for high-temperature ethanol-production processes in thermophilic bacteria.

However, our current knowledge of bacterial PDCs is extremely limited, as so far only six bacterial PDCs have been described. These are PDCs from Acetobacter pasteurianus (ApPDC; PDB entry 2vbi; D. Gocke, C. L. Berthold, G. Schneider \& M. Pohl, unpublished work), Gluconoacetobacter diazotrophicus (GdPDC; PDB entry 4cok; van Zyl, Schubert et al., 2014), Gluconobacter oxydans (GoPDC; van 
Zyl, Taylor et al., 2014) and Zymobacter palmae (ZpPDC; PDB entry 5euj; Buddrus et al., 2016), the extensively studied Zymomonas mobilis PDC (ZmPDC; PDB entries 1zpd, 2wva, 2wvg, 2wvh, 3oei and 4zp1; Dobritzsch et al., 1998; Meyer et al., 2010; Pei et al., 2010; Wechsler et al., 2015) and a PDC from Sarcina ventriculi (SvPDC; Lowe \& Zeikus, 1992), which is the only Gram-positive species known to possess a PDC.

One approach to open up new opportunities for bacterial PDCs adapted to a variety of environments is to exploit the diversity provided by evolution. Ancestral sequence reconstruction (ASR), first envisaged by Pauling \& Zuckerkandl (1963), is a method of computational molecular evolution to infer extinct ancestral protein sequences, which can then be synthesized and experimentally characterized (for a comprehensive review of state-of-the-art ASR methods, see Merkl \& Sterner, 2016). ASR explores a sequence space that has already been screened over evolutionary time spans, thus reducing the nonfunctional space that would otherwise be included in protein libraries generated by random mutagenesis, for example. Thus, ASR has an advantage over random or purely computational approaches in that it limits the 'design space' to proteins that are properly folded and have a demonstrable activity (Cole \& Gaucher, 2011; Hobbs et al., 2012).

Many enzymes reconstructed using ASR show higher thermostability and kinetic activity when compared with modern enzymes (Akanuma et al., 2013; Groussin et al., 2015; Hobbs et al., 2012, 2015; Perez-Jimenez et al., 2011; Risso et al., 2013). Thus, ASR is a promising protein tool for the purpose of generating enzymes and proteins with favourable thermal properties.

In this study, we employed ASR to infer an ancestral bacterial PDC designated ANC27. Here, we present the crystal structure of the inferred ancestral PDC ANC27 at $3.5 \AA$ resolution, show that the enzyme is fully functional and compare it with known extant bacterial PDCs.

\section{Materials and methods}

\subsection{Ancestral sequence reconstruction}

2.1.1. PDC sequences. The ASR input was 25 amino-acid sequences of extant bacterial PDCs retrieved from GenBank or identified through a $B L A S T$ search based on $>50 \%$ aminoacid sequence identity over $>90 \%$ coverage to the reference ZpPDC (PDB entry 5euj). Full details of the strains used and the protein/gene accession numbers can be found in Supplementary Table S1.

2.1.2. Phylogenetic analysis and node-age estimates. Following the approach used by Hobbs et al. (2012), the PDC amino-acid sequences were aligned using Geneious (v.R7 7.1.7; Kearse et al., 2012) with the MUSCLE alignment option (eight iterations) and then refined using Gblocks (Castresana, 2000). ProtTest (Abascal et al., 2005) was used to determine the most appropriate model of amino-acid evolution (WAG + $\mathrm{F}+$ ). This model and the Gblocks alignment were used in PhyML (v.3.0; Guindon et al., 2010) to build a phylogenetic guide tree based on maximum-likelihood (ML) phylogenies. This guide tree, together with the MUSCLE alignment, was then implemented in PRANK (Löytynoja \& Goldman, 2010) to generate an amino-acid alignment based on phylogenetic information. The resulting alignment was analysed using GARLI 2.0 (Zwickl, 2010) while implementing the WAG model of evolution to find the best ML tree based on loglikelihood scores (see Supplementary Fig. S1). GARLI was also used to bootstrap the tree using 1024 replicates. This tree was rooted to the outgroup (PDC sequences with $<60 \%$ amino-acid identity to ZpPDC, in this case SvPDC with $31 \%$ and Ktedonobacter racemifer PDC with 52\%) in Geneious.

The best ML GARLI tree was aged using r8s (v.1.8; Sanderson, 2003; available at http://loco.biosci.arizona.edu/ $\mathrm{r} 8 \mathrm{~s} /$ ). The point of divergence of proteobacteria and firmicutes (3.19 billion years ago) was used as the calibration point, based on a robust prokaryotic phylogeny study using 32 protein sequences and molecular-divergence times estimated from geological calibration points (Battistuzzi et al., 2004). The resulting tree was visualized in FigTree (v.1.4.2; available at http://tree.bio.ed.ac.uk/software/figtree/). See Supplementary Fig. S2 for the maximum-likelihood chronogram.

2.1.3. Ancestral reconstruction. Three different methods of ancestral inference (amino-acid, nucleotide and codon inference) were performed using the PAML software (v.4.3; Yang, 2007) under the ML criterion. $P d c$ sequences were collated in Geneious and manually curated to include gaps as determined by the $P R A N K$ amino-acid sequence alignment. Nucleotidesequence inference in $B A S E M L$ used the REV/GTR nucleotide-substitution rate model (with rate variation $+\mathrm{I}$ and $+\mathrm{G})$, which was determined to be the most appropriate model of evolution in JModelTest (v.2.1.5; Posada, 2008). GTR was also employed for codon inference in CODEML, while for amino-acid inference the WAG model was used. Ancestral gaps were inferred in $P R A N K$ using the $P R A N K$ amino-acid alignment and the best ML GARLI tree. Taken together, the inferred sequences for nodes of interest were compiled by generating a consensus sequence from all methods of inference. Any ambiguities in the resulting consensus amino-acid sequence were resolved taking the following into consideration: (i) physiochemical properties, (ii) the structural environment, (iii) the most common residues present in extant sequences and (iv) the residue as predicted by the codoninference method (considered to be the most robust method; Hobbs et al., 2012).

\subsection{Macromolecule production}

The amino-acid sequence generated through ASR was backtranslated and synthesized by Eurofins, including XbaI and XhoI restriction sites for cloning into pET-28a(+) (Novagen). The resulting construct added a thrombin cleavage site, a $3 \times$ glycine linker and a hexahistidine tag to the C-terminus of ANC27, and was transformed into Escherichia coli BL21 (DE3) cells for expression.

For overexpression, the $E$. coli cells were grown in autoinducing Overnight Express TB (Novagen) medium 
Table 1

Details relating to the production of ANC27 PDC.

Source organism
DNA source
Expression vector
Expression host
Complete amino-acid sequence
of the construct produced $\dagger$

\begin{abstract}
Inferred ancestral sequence
Ancestral sequence reconstruction; see Supporting Information for the DNA sequence

pET-28a(+) (Novagen)

E. coli BL21 (DE3)

MTYTVGHYLATRLAQIGLKHHFAVAGDYNL

VLLDQLLKNKDLEQVYCCNELNCGFSAE GYARANGVGAAVVTFSVGALSAFNAIGG

AYAENLPVILISGAPNTNDHGSGHILHH TIGTTDYGYQLEMAKQI TCAAVS ITHAE DAPALIDHAIRTALREKKPAYIEIACNV AAQPCARPGPVSALLNEPTSDEETLKAA VEAALDFIEKREKPVLLVGGKLRAAGAE EAVVELADALGCAVATMAAAKSFFPEDH PGYVGTYWGEVSSPGVEE IVDWADG I IC LGPVFNDYSTVGWTAWPKGENVVLVDPH HITVGGEEFTGIHLKDFLTALTERVPKK DATLDQFKARVGKPAAEKVPAADPNAPI TRAELCRQIQGLLNPNTTLIAETGDSWE NAMRMKLPHGARVELEMQWGHIGWSVPA TFGYAVAEPERRNVLMVGDGSFQLTAQE VAQMVRRKLPI I IFLINNRGYTIEVKIH DGPYNNIKNWDYAGLMEVFNAEDGKGLG LKATTGGELAEAIKKALAHREGPTLIEC VIDRDDCTPELVTWGKKVATANARPPQA ILVPRGSGGGLEHHHHHH
\end{abstract}

$\dagger$ The C-terminal tag containing a thrombin cleavage site, a $3 \times$ Gly linker and a hexahistidine tag is underlined.

supplemented with $100 \mu \mathrm{g} \mathrm{ml}^{-1}$ kanamycin and $5 \mathrm{mM}$ thiamine chloride at $303 \mathrm{~K}$ for $16 \mathrm{~h}$ with shaking at $220 \mathrm{rev} \mathrm{min}^{-1}$.

The cells were harvested by centrifugation $(4000 g, 277 \mathrm{~K})$ and resuspended in His-bind buffer $(20 \mathrm{~m} M$ Tris $\mathrm{pH} 8$, $300 \mathrm{~m} M \mathrm{NaCl}, 20 \mathrm{~m} M$ imidazole). The cells were lysed by sonication on ice and the insoluble debris was removed by centrifugation $(17000 \mathrm{~g}, 277 \mathrm{~K}, 30 \mathrm{~min})$. The supernatant was loaded onto a $5 \mathrm{ml}$ HisTrap HP (GE Healthcare) column for nickel-affinity chromatography. The column was washed with ten column volumes of His-bind buffer before eluting the protein with increasing concentrations of His-elute buffer (20 $\mathrm{m} M$ Tris $\mathrm{pH} 8,300 \mathrm{~m} M \mathrm{NaCl}, 1 M$ imidazole) on an ÄKTAexplorer FPLC system (GE Healthcare), monitoring the eluted protein at $280 \mathrm{~nm}$.

The eluted protein was buffer-exchanged into $50 \mathrm{~m} M$ MES

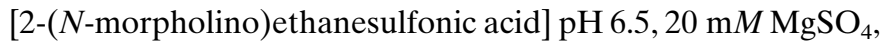
$3 \mathrm{~m} M$ TPP using a Superdex 200 10/300 GL gel-filtration column (GE Healthcare) on an ÄKTAexplorer FPLC system and purified to $>95 \%$ homogeneity as determined by $12 \%$ SDS-PAGE analysis.

The enzyme-activity assays were performed as described by Raj et al. (2002), with the reaction mixture consisting of $0.15 \mathrm{~m} M \mathrm{NADH}, 20 \mathrm{~m} M \mathrm{MgSO}_{4}, 3 \mathrm{~m} M$ thiamine pyrophosphate (TPP), $29 \mathrm{mM}$ pyruvate and $10 \mathrm{U}$ Saccharomyces cerevisiae alcohol dehydrogenase (Sigma-Aldrich) in $50 \mathrm{~m} M$ MES pH 6.5 at $303 \mathrm{~K}$. The reduction in NADH concentration was followed spectrophotometrically at $340 \mathrm{~nm}$. Kinetic properties were analysed using the nonlinear-fit model from the enzymekinetics module in SigmaPlot (Systat Software). The temperature optimum was determined as described by Gocke et al. (2009) by following the depletion of pyruvate at $320 \mathrm{~nm}$.
Table 2

Crystallization conditions.

\begin{tabular}{ll}
\hline Method & Hanging-drop vapour diffusion \\
Plate type & 24 -well \\
Temperature $(\mathrm{K})$ & 291 \\
Protein concentration $\left(\mathrm{mg} \mathrm{ml}^{-1}\right)$ & 4.5 \\
Buffer composition of protein & $50 \mathrm{~m} M$ MES pH 6.5, 20 mM MgSO \\
$\quad$ solution & $3 \mathrm{~m} M$ TPP, $2 \mathrm{~m} M$ pyruvate \\
Composition of reservoir solution & $0.15 M$ sodium citrate pH 5.5, \\
& $14 \%(w / v)$ PEG 3350 \\
Volume and ratio of drop & $2 \mu 1,1: 1$ \\
Volume of reservoir (ml) & 0.4 \\
\hline
\end{tabular}

The denaturation temperature was determined using SYPRO Orange dye in thermal shift assays as described in Huynh \& Partch (2015).

Information relating to the production of ANC27 PDC is summarized in Table 1.

\subsection{Crystallization}

Crystals of the purified ANC27 were obtained using the hanging-drop vapour-diffusion method at $291 \mathrm{~K}$. The same approach was used for the crystallization of ANC27 as had been used for earlier PDC structures (Buddrus et al., 2016). A screen assessing $4 \times 96$ conditions was carried out in the presence of ligand, and those conditions that resulted in crystal growth were followed up with multiple fine screens to optimize conditions for crystal growth. These efforts did not result in crystals of better diffraction quality. Multiple crystals were screened for diffraction at the home source, and the crystals with the 'best' diffraction were further screened at the Australian Synchrotron (AS) for data collection. Following these assessments, further optimization of conditions was carried out; however, the crystal quality did not improve. The data were collected from crystals grown from purified ANC27 that had been incubated with $2 \mathrm{~m} M$ pyruvate for $30 \mathrm{~min}$ at room temperature ( $293 \mathrm{~K}) .2 \mu \mathrm{l}$ drops of the enzyme/pyruvate solution mixed in a 1:1 ratio with crystallization solution [0.15 $M$ sodium citrate $\mathrm{pH} 5.5,14 \%(w / v)$ PEG 3350] were placed onto cover slips and equilibrated against $400 \mu \mathrm{l}$ reservoir solution. Crystals were looped out and soaked in cryoprotectant $[10 \%(w / v)$ glycerol added to the crystallization buffer] before flash-cooling and storage in liquid nitrogen. Crystallization information is summarized in Table 2.

\subsection{Data collection and processing}

X-ray diffraction data were collected to $3.5 \AA$ resolution on the MX2 beamline at the Australian Synchrotron, Melbourne, Australia. iMosflm (Battye et al., 2011), AIMLESS (Evans \& Murshudov, 2013) and BALBES (Long et al., 2008) were used for data reduction, data scaling and phasing, respectively. The crystal belonged to space group $P 3_{2} 21$, with unit-cell parameters $a=b=108.333, c=322.65 \AA$, and contained two dimers (tetramer halves) in the asymmetric unit. Data-collection and processing statistics are summarized in Table 3. 
Table 3

Data collection and processing.

Values in parentheses are for the outer shell.

\begin{tabular}{ll}
\hline Diffraction source & Beamline MX2, AS \\
Wavelength $(\AA)$ & 0.9537 \\
Temperature $(\mathrm{K})$ & 100.0 \\
Detector & ADSC Quantum 315r CCD \\
Crystal-to-detector distance $(\mathrm{mm})$ & 450 \\
Rotation range per image $\left(^{\circ}\right)$ & 1 \\
Total rotation range $\left(^{\circ}\right)$ & 101 \\
Exposure time per image $(\mathrm{s})$ & 1 \\
Space group & $P 3_{2} 21$ \\
$a, b, c(\AA)$ & $108.33,108.33,322.65$ \\
Mosaicity $\left(^{\circ}\right)$ & 1.09 \\
Resolution range $(\AA)$ & $93.82-3.50(3.71-3.50)$ \\
Total No. of reflections & $125993(20708)$ \\
No. of unique reflections & $28526(4512)$ \\
Completeness $(\%)$ & $99.5(99.9)$ \\
Multiplicity & $4.4(4.6)$ \\
$\langle I / \sigma(I)\rangle \dagger$ & $5.6(1.5)$ \\
$R_{\text {r.i.m. }}$ & $0.142(0.565)$ \\
Mn $(I)$ half-set correlation $\mathrm{CC}_{1 / 2}$ & $0.965(0.422)$ \\
Overall $B$ factor from Wilson plot $\left(\AA^{2}\right)$ & 53.8
\end{tabular}

$\dagger$ The resolution at which $I / \sigma(I)$ falls below 2.0 is $3.79 \AA$; the cutoff at $3.5 \AA$ was chosen as the 'maximum resolution' by AIMLESS using $\mathrm{CC}_{1 / 2}$.

\subsection{Structure solution and refinement}

The structure was solved by molecular replacement in $B A L B E S$ using ZmPDC (PDB entry 2wvg; $71 \%$ amino-acid identity) as the starting model. The structure was refined by iterative cycles of manual building and modelling in Coot (Emsley et al., 2010) and refinement in REFMAC5 (CCP4 suite; Winn et al., 2011; Potterton et al., 2018; Murshudov et al., 2011) and PHENIX (Adams et al., 2010). Refinement in REFMAC5 was restricted with noncrystallographic symmetry (NCS) local restraints and TLS (translation, liberation, screw rotation) restraints, and included jelly-body and isotropic temperature factors under automatic weighting. The NCS restraints and TLS parameters were also applied in PHENIX. The quality of the final model was checked using MolProbity (Chen et al., 2010). The structure has been submitted to the Protein Data Bank and assigned PDB entry 5npu. Table 4 summarizes the structure-solution and refinement statistics.

\section{Results and discussion}

\subsection{ASR produced a fully functional bacterial PDC}

ANC27 (with an inferred age of 1248 million years; Supplementary Fig. S2) was reconstructed using ASR. The three methods of inference (amino-acid, nucleotide and codon inference) resulted in $<3 \%$ ambiguous sites, which were resolved as described in $\$ 2.1 .3$. The average posterior probability at each site was 0.6946 (confidence scores for aminoacid, codon and nucleotide inference were $0.89443,0.43704$ and 0.77432 , respectively). The final amino-acid sequence showed an amino-acid identity of $<79 \%$ compared with all extant sequences used in the inference (Supplementary Table S2).

Investigation of the kinetic properties of ANC27 showed a $V_{\max }$ of $536 \pm 13 \mathrm{U} \mathrm{mg}^{-1}$ and a $K_{\mathrm{m}}$ for pyruvate of
Table 4

Structure solution and refinement.

Values in parentheses are for the outer shell.

\begin{tabular}{|c|c|}
\hline Resolution range $(\AA)$ & $81.1-3.5(3.62-3.50)$ \\
\hline Completeness (\%) & $99.4(100)$ \\
\hline$\sigma$ Cutoff & $F>1.330 \sigma(F)$ \\
\hline No. of reflections, working set & $27079(2690)$ \\
\hline No. of reflections, test set & $1358(122)$ \\
\hline Final $R_{\text {cryst }}$ & $0.246(0.3671)$ \\
\hline Final $R_{\text {free }}$ & $0.319(0.4482)$ \\
\hline \multicolumn{2}{|l|}{ No. of non-H atoms } \\
\hline Protein & 16540 \\
\hline Ligand & $\begin{array}{l}115 \text { [TPP, } 26 \text { atoms each; Mg, } \\
1 \text { atom each; PEG, } 7 \text { atoms] }\end{array}$ \\
\hline Solvent & 1 \\
\hline Total & 16656 \\
\hline \multicolumn{2}{|l|}{ R.m.s. deviations } \\
\hline Bonds $(\AA)$ & 0.007 \\
\hline Angles $\left({ }^{\circ}\right)$ & 0.752 \\
\hline \multicolumn{2}{|l|}{ Average $B$ factors $\left(\AA^{2}\right)$} \\
\hline Protein & 87.115 \\
\hline Ligand & 85.826 \\
\hline \multicolumn{2}{|l|}{ Ramachandran plot } \\
\hline Most favoured (\%) & 92.75 \\
\hline Allowed (\%) & 6.88 \\
\hline
\end{tabular}

$3.6 \pm 0.3 \mathrm{~m} M$. The $K_{\mathrm{m}}$ is comparable to data from previously characterized extant bacterial PDCs (Buddrus et al., 2016). However, the $V_{\max }$ is higher than for any reported bacterial PDC. The highest previously reported $V_{\max }$ was $181 \mathrm{U} \mathrm{mg}^{-1}$, determined for Z. mobilis PDC (at pH 6 and $30^{\circ} \mathrm{C}$; BringerMeyer et al., 1986). Furthermore, the $k_{\text {cat }}$ of ANC27 is the highest reported for bacterial PDCs at $580 \mathrm{~s}^{-1} \cdot k_{\mathrm{cat}} / K_{\mathrm{m}}$ is very similar to those for GoPDC and GdPDC (Table 5).

Comparing the thermal properties of ANC27 with those of extant PDCs (summarized in Table 6), ANC27 is one of the least thermostable and thermoactive bacterial PDCs currently known, only surpassing SvPDC, with which it shares only $33 \%$ amino-acid sequence identity.

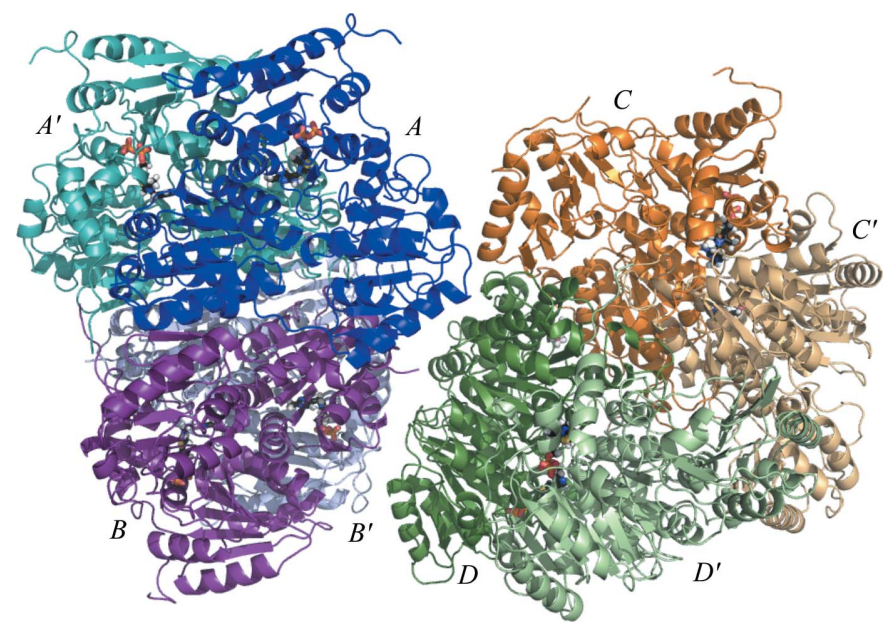

Figure 1

Cartoon representation of ANC27. The four monomers coloured in darker shades of blue (labelled $A$ ), purple $(B)$, green $(D)$ and orange $(C)$ are present in the asymmetric unit in the final model, while the four outermost monomers coloured pale beige $\left(C^{\prime}\right)$, pale green $\left(D^{\prime}\right)$, pale violet $\left(B^{\prime}\right)$ and pale teal $\left(A^{\prime}\right)$ are generated through symmetry. TPP molecules bound between the PYR domain of one monomer and the PP domain of the other are represented as sticks coloured by atom. 
Table 5

Kinetic properties of known bacterial PDCs in order of increasing thermostability (assay buffer at pH 6.5 unless indicated otherwise).

\begin{tabular}{|c|c|c|c|c|c|c|c|}
\hline & SvPDC & ANC27 & GdPDC & GoPDC & $\mathrm{ZmPDC}$ & ApPDC & ZpPDC \\
\hline Amino-acid identity (\%) & 33 & Reference & 74 & 66 & 71 & 67 & 69 \\
\hline Kinetics & Sigmoidal & Michaelis-Menten & Michaelis-Menten & Michaelis-Menten & Michaelis-Menten & Michaelis-Menten & Michaelis-Menten \\
\hline$V_{\max }\left(\mathrm{U} \mathrm{mg}^{-1}\right)$ & $45 \dagger$ & $536 \pm 13$ & $43(\mathrm{pH} \mathrm{7}) \ddagger$ & $125(\mathrm{pH} 7) \dagger$ & $121 \S$ & $110 \pm 2 \S$ & $\begin{array}{l}165 \pm 3 \\
116 \pm 2 \S\end{array}$ \\
\hline$K_{\mathrm{m}}\left(S_{0.5}\right)(\mathrm{m} M)$ & $5.7 \dagger$ & $3.6 \pm 0.3$ & $1.2(\mathrm{pH} \mathrm{7}) \ddagger$ & $2.8(\mathrm{pH} 7) \dagger \dagger$ & $1.3 \S$ & $2.8 \pm 0.2 \S$ & $\begin{array}{l}0.67 \pm 0.05 \\
2.5 \pm 0.2 \S\end{array}$ \\
\hline$k_{\text {cat }}\left(\mathrm{s}^{-1}\right)$ & $412+\%$ & 580 & NA & $125(\mathrm{pH} 7)+\dagger$ & $486 \dagger$ & $341-508 \dagger$ & $341-508 \dagger$ \\
\hline$k_{\text {cat }} / K_{\mathrm{m}}\left(M^{-1} \mathrm{~s}^{-1}\right)$ & $3.2 \times 10^{4}+\neq$ & $1.6 \times 10^{5}$ & $1.4 \times 10^{5}(\mathrm{pH} 7.0)$ & $1.6 \times 10^{5}+\dagger$ & $4.4 \times 10^{5} \S \S$ & $1.3 \times 10^{6}(\mathrm{pH} 5.0) \ddagger$ & $1.4 \times 10^{6}(\mathrm{pH} 6.0)$ \\
\hline GenBank protein ID & AAL18557.1 & NA & AIG13066.1 & AHB37781.1 & AAA27696.2 & AAM21208.1 & AAM49566.1 \\
\hline R.m.s.d. $(\AA)$ & NA & Refere & 0.83 & NA & 0.76 & 0.87 & 0.82 \\
\hline$Q$ scores & NA & Reference & 0.85 & NA & 0.86 & 0.84 & 0.86 \\
\hline
\end{tabular}

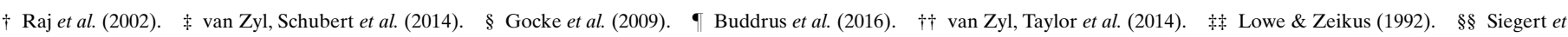
al. (2005).

Table 6

Thermal properties of known bacterial PDCs in order of increasing thermostability.

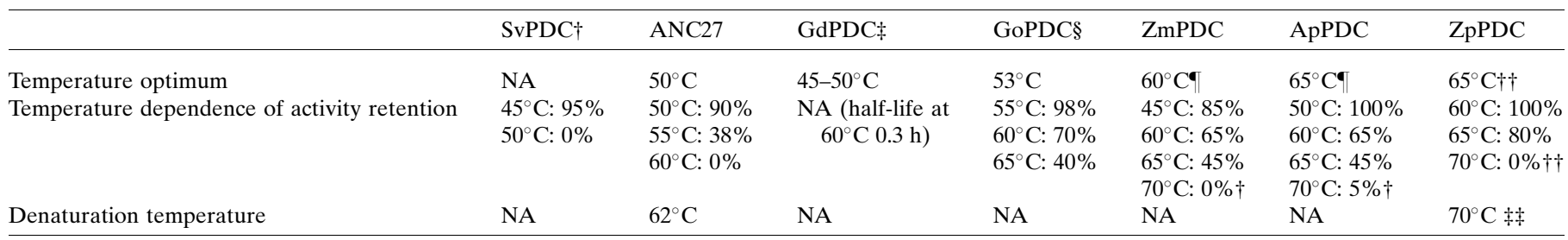

$\dagger$ Raj et al. (2002). \$ van Zyl, Schubert et al. (2014). § van Zyl, Taylor et al. (2014). @ Gocke et al. (2009). †† Buddrus et al. (2016).

\subsection{Crystal structure}

The crystal structure of ANC27 was determined by molecular replacement using ZmPDC (PDB entry 2wvg; 71\% amino-acid identity) as the starting model, with the correct solution confirmed by the presence of electron density for TPP, which was omitted from the search model. As described

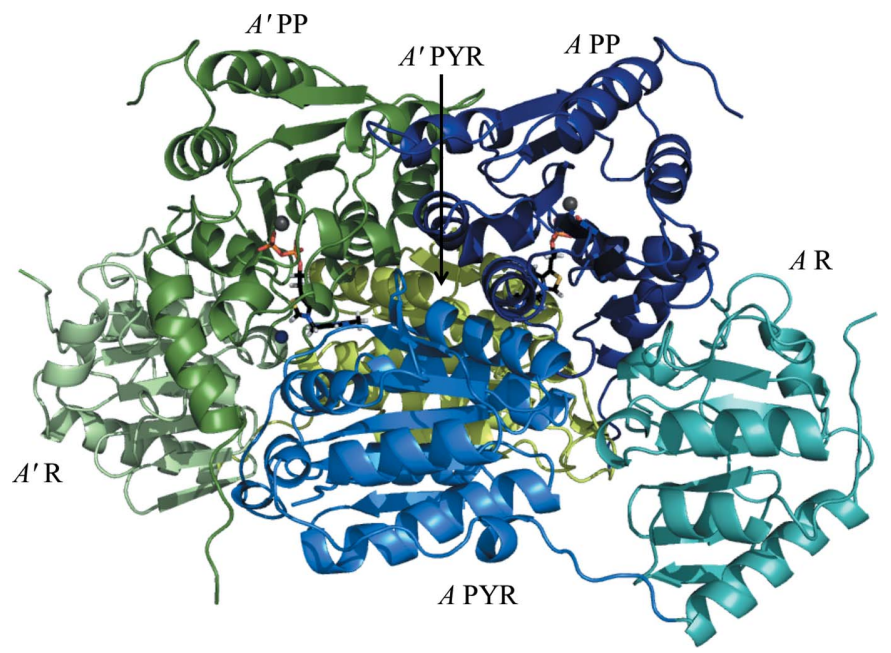

Figure 2

Cartoon representation of an ANC27 dimer. The monomers are coloured by domain. Monomer $A$ is coloured blue, with the PYR domain (residues 2-191) in light blue, the $\mathrm{R}$ domain (residues 191-361) in teal and the PP domain (residues $362-563$ ) in dark blue. The second monomer $\left(A^{\prime}\right)$ is coloured cyan, with the PYR domain in green-cyan and indicated by an arrow, the $\mathrm{R}$ domain in pale cyan and the PP domain in dark teal. TPP molecules are represented as sticks coloured by atom. Magnesium ions are represented as grey spheres, while water is shown as a blue sphere. for extant bacterial PDCs, the quaternary structure of this inferred ancestral PDC is a homotetramer or dimer of dimers (Fig. 1). The domains can be assigned as follows: amino acids 1-191, PYR (pyrimidine binding, with 177-191 linker); 192361, R (regulatory, with 341-361 linker); 362-563, PP (pyrophosphate binding) (Fig. 2). A dimer forms when TPP binds across the two subunits, with the pyrophosphate group binding to the PP domain from one subunit and the pyrimidine ring binding to the PYR domain from the second subunit, thus forming two active sites in the dimer. The final model comprises four chains of 563 amino acids with a molecular mass of $60.6 \mathrm{kDa}$, each with a TPP and an $\mathrm{Mg}^{2+}$ ion, together with one water in the active site of chain $A$ and one PEG molecule between chains $A$ and $C$. The final model contains four monomers in the asymmetric unit, each comprising part of a dimer formed with a symmetry-related monomer, with the dimers coming together in a less tight interaction to form dimer-of-dimers tetramers. A full tetramer would have a surface area of $65130 \AA^{2}$.

Superposition of the four monomers yielded an average root-mean-square deviation (r.m.s.d.) of $0.213 \AA$, indicating that the structures are very similar.

Overall, the electron-density map was of good quality, apart from the N-terminal methionine and the loop 340-360, which is the exposed linker between the $\mathrm{R}$ and PP domains. These regions seem to be very flexible and have not been included in the final model (more specifically chain $A$ residues 344-358, chain $B$ residues $343-359$, chain $C$ residues $343-357$, chain $D$ residues 340-358 and the $\mathrm{N}$-terminal methionine in all chains). Compared with other crystal structures of bacterial PDCs 
Table 7

Comparison of the flexibility of bacterial PDCs in order of increasing thermostability.

\begin{tabular}{|c|c|c|c|c|c|}
\hline & $\begin{array}{l}\text { ANC27 (PDB } \\
\text { entry 5npu) }\end{array}$ & $\begin{array}{l}\text { GdPDC (PDB } \\
\text { entry 4cok) }\end{array}$ & $\begin{array}{l}\mathrm{ZmPDC}(\mathrm{PDB} \\
\text { entry 1zpd) }\end{array}$ & $\begin{array}{l}\text { ApPDC (PDB } \\
\text { entry 2vbi) }\end{array}$ & $\begin{array}{l}\text { ZpPDC (PDB } \\
\text { entry 5euj) }\end{array}$ \\
\hline Overall $B$ factor from Wilson plot $\left(\AA^{2}\right)$ & 53.8 & 14.5 & 10.4 & & \\
\hline \multicolumn{6}{|l|}{ Average $B$ factors $\left(\AA^{2}\right)$} \\
\hline Overall & 51.1 & 15.3 & 14.9 & 42.7 & 26.6 \\
\hline Protein & 87.1 & 13.5 & 13.2 & 42.4 & 26.9 \\
\hline Ligand & 85.8 & 22.6 & 14.4 & 38.2 & 24.6 \\
\hline $\mathrm{TPP}$ & 85.0 & 14.2 & 12.8 & 38.3 & 23.5 \\
\hline $\mathrm{R}-\mathrm{PP}$ linker loop & $\begin{array}{c}\text { First and last residue } \\
\text { present: } 68.4,70.5\end{array}$ & 14.9 & 23.6 & 43.1 & 49.6 \\
\hline
\end{tabular}

Table 8

Comparison of the interface areas of bacterial PDCs in order of increasing thermostability as calculated using PDBePISA (Krissinel \& Henrick, 2007).

\begin{tabular}{|c|c|c|c|c|c|}
\hline & $\begin{array}{l}\text { ANC27 (PDB } \\
\text { entry } 5 \mathrm{npu})\end{array}$ & $\begin{array}{l}\text { GdPDC (PDB } \\
\text { entry 4cok) }\end{array}$ & $\begin{array}{l}\mathrm{ZmPDC}(\mathrm{PDB} \\
\text { entry 1zpd) }\end{array}$ & $\begin{array}{l}\text { ApPDC (PDB } \\
\text { entry 2vbi) }\end{array}$ & $\begin{array}{l}\text { ZpPDC (PDB } \\
\text { entry 5euj) }\end{array}$ \\
\hline Interface area between monomers within a functional dimer $\left(\AA^{2}\right)$ & 3947.4 & 3749.8 & $4144.5(4387 \dagger)$ & 3761.3 & 3813.1 \\
\hline Percentage of total monomer surface & 18.06 & 17.83 & $18.39(19.4 \dagger)$ & 16.95 & 17.08 \\
\hline Interaction area between two functional dimers to form a tetramer $\left(\AA^{2}\right)$ & 1399.4 & 1851.4 & $2489.2(4405 \dagger)$ & 2840 & 2912.16 \\
\hline Tetramer interface as a percentage of total surface of one dimer & 6.4 & 8.79 & $11.03(12.1 \dagger)$ & 12.78 & 13.06 \\
\hline
\end{tabular}

$\dagger$ Dobritzsch et al. (1998)

Table 9

Comparison of the interactions within interfaces of bacterial PDC structures in order of increasing thermostability; interactions were determined using PDBePISA (Krissinel \& Henrick, 2007).

\begin{tabular}{|c|c|c|c|c|c|c|c|c|c|c|}
\hline \multirow[b]{2}{*}{ Interactions on interfaces } & \multicolumn{2}{|c|}{$\begin{array}{l}\text { ANC27 } \\
\text { (PDB entry 5npu) }\end{array}$} & \multicolumn{2}{|c|}{$\begin{array}{l}\text { GdPDC } \\
\text { (PDB entry 4cok) }\end{array}$} & \multicolumn{2}{|c|}{$\begin{array}{l}\text { ZmPDC } \\
\text { (PDB entry 1zpd) }\end{array}$} & \multicolumn{2}{|c|}{$\begin{array}{l}\text { ApPDC } \\
\text { (PDB entry 2vbi) }\end{array}$} & \multicolumn{2}{|c|}{$\begin{array}{l}\text { ZpPDC } \\
\text { (PDB entry 5euj) }\end{array}$} \\
\hline & $\begin{array}{l}\text { Hydrogen } \\
\text { bonds }\end{array}$ & $\begin{array}{l}\text { Salt } \\
\text { bridges }\end{array}$ & $\begin{array}{l}\text { Hydrogen } \\
\text { bonds }\end{array}$ & $\begin{array}{l}\text { Salt } \\
\text { bridges }\end{array}$ & $\begin{array}{l}\text { Hydrogen } \\
\text { bonds }\end{array}$ & $\begin{array}{l}\text { Salt } \\
\text { bridges }\end{array}$ & $\begin{array}{l}\text { Hydrogen } \\
\text { bonds }\end{array}$ & $\begin{array}{l}\text { Salt } \\
\text { bridges }\end{array}$ & $\begin{array}{l}\text { Hydrogen } \\
\text { bonds }\end{array}$ & $\begin{array}{l}\text { Salt } \\
\text { bridges }\end{array}$ \\
\hline Dimer interface & 66 & 15 & 63 & 13 & $76(66 \dagger)$ & $14(7 \dagger)$ & 61 & 16 & 73 & 12 \\
\hline Major tetramer interface (neighbour $\ddagger$ ) & 8 & 0 & 17 & 9 & $29(64 \dagger)$ & $8(25 \dagger)$ & 34 & 14 & 31 & 24 \\
\hline Minor tetramer interface (diagonal $\ddagger$ ) & 1 & 0 & 4 & 3 & 6 & 2 & 4 & 0 & 2 & 0 \\
\hline TPP pyrimidine ring & 10 & 0 & 10 & 0 & 12 & 0 & 10 & 0 & 11 & 0 \\
\hline
\end{tabular}

$\dagger$ Dobritzsch et al. (1998). \$ Neighbour, interactions between neighbouring monomers; diagonal, interactions between monomers diagonally across tetrameric centre.

ANC27 has much higher average $B$ factors (Table 7). However, in all the PDCs the exposed linker between the R and PP domains is more mobile than the molecule as a whole.

The final model was refined to an $R$ factor of $24.6 \%$ and an $R_{\text {free }}$ value of $31.86 \%$ using data between 81.1 and $3.5 \AA$ resolution. $92.75 \%$ of the residues are located in favoured regions of the Ramachandran plot and a further $6.88 \%$ are in allowed regions.

\subsection{Structural comparison}

It has been noted previously that a trend for increasing tetramer interface areas and increasing numbers of interactions at interfaces (i.e. forming hydrogen bonds or salt bridges) can be observed as the thermoactivity and thermostability of the PDCs increase (Buddrus et al., 2016). The interactions between the monomers within a dimer, however, are constant across the thermostability range $\left(45-65^{\circ} \mathrm{C}\right)$. This observation is supported by the data obtained from ANC27. Table 8 summarizes the interface areas and Table 9 summarizes the numbers of interactions made between different interfaces.
Using PROMALS3D (Pei et al., 2010) to generate a structure-based alignment, conserved regions were analysed. Domains are well conserved, but linker regions, especially the linker region between the $\mathrm{R}$ and $\mathrm{PP}$ domains, are less so. The R-PP linker appears to be extended in ANC27 (Supplementary Fig. S3). However, the diversity displayed by the extant PDCs in the length, sequence and structural positioning of this linker region suggests that it may play a subtle role in the thermostability and thermoactivity differences observed between these PDCs, as previously mentioned by van Zyl, Schubert et al. (2014). GdPDC is one of the least thermostable PDCs, but has one of the shortest linker regions. In ZmPDC this linker is four amino acids longer than the linker in the most thermostable PDC, ZpPDC. The ZpPDC linker is five amino acids shorter than the linker in ANC27.

\subsection{The active site and TPP binding}

Four monomers form a tetramer with pseudo-222 symmetry and create four active sites. There is good-quality electrondensity evidence for a TPP molecule and an $\mathrm{Mg}^{2+}$ ion in each of the four chains in the structure. Each TPP binds as indicated by the domain nomenclature, with the pyrophosphate group 
binding to the PP domain of one monomer and the pyrimidine ring binding to the PYR domain of a second monomer in a dimer, thus creating the active site in a narrow cleft at the interfaces between the two monomers (Fig. 2).

As noted in PDCs from extant bacteria, TPP is held in a V shape by Ile416 from the first monomer (Fig. 3 and Supplementary Fig. S4). The $\mathrm{O}$ atoms of the pyrophosphate form hydrogen bonds to the backbone amide groups of Ile473 and Thr472, while the $\mathrm{N} 1$ atom on the pyrimidine ring forms a hydrogen bond to Glu50 (from the second monomer). These interactions are essential for the catalytic mechanism (Pei et al., 2010). Tyr471 and Glu474 from one monomer are involved in the binding of the pyrophosphate, while Thr72 from the second monomer is involved in TPP binding around the pyrimidine ring.

Through interactions with Asp27 and Glu474, a water molecule supports the active-site arrangement. This has also been observed in extant PDCs, even in the absence of substrate, and is thought to play a pivotal role in the organization of the substrate complex and of the hydrogen-bond network (Pei et al., 2010).

The $\mathrm{Mg}^{2+}$ ion anchors the pyrophosphate group of the TPP and forms interactions with the two $\mathrm{O}$ atoms on the diphosphate group of the TPP, the side-chain O atoms of Asp441 and Asn467 and the main-chain O atom of Gly470. In other PDC structures a water molecule completes the coordination sphere, but in this low-resolution structure this cannot be defined. In all other respects the conformation of TPP and the $\mathrm{Mg}^{2+}$ ion are the same in this and the other PDC structures (Supplementary Fig. S4).

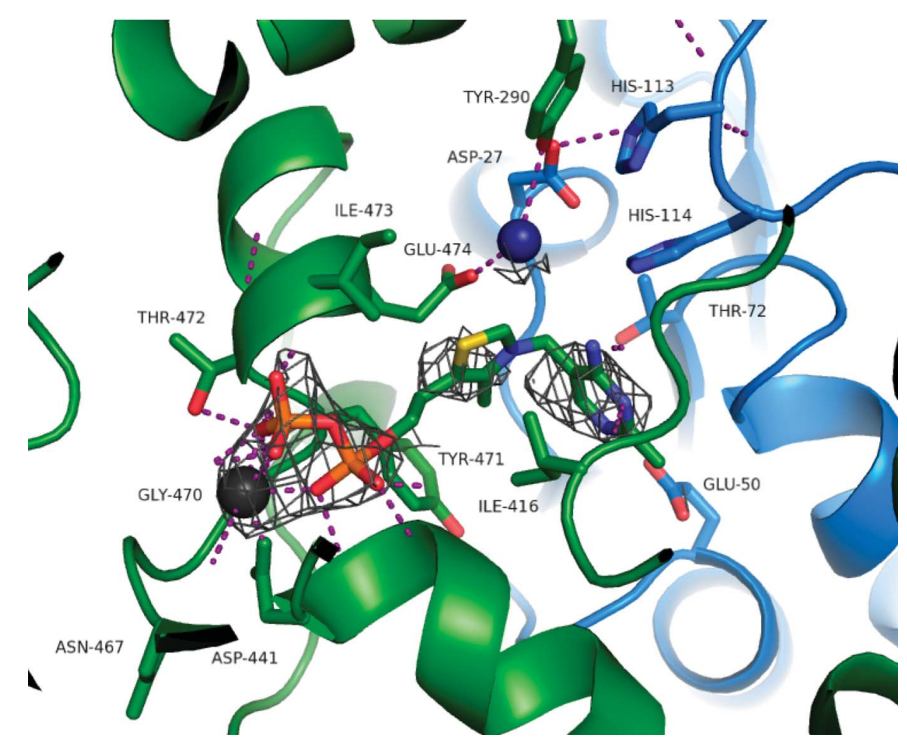

Figure 3

Cartoon and stick depiction of the active site. Residues of one monomer are coloured in green; residues of the other monomer are coloured in blue. The magnesium (dark grey) and water (dark blue) molecules are represented as spheres. The TPP for the green chain is shown as a stick model and coloured by atom. Hydrogen bonds are depicted as magenta dotted lines. The map shown around TPP and magnesium is a simulatedannealing composite OMIT map contoured at $1.5 \sigma$.

\subsection{Ancestral reconstruction}

This ASR study used an unconventional approach in only using mesophilic species as the inference input to reconstruct a bacterial enzyme, as bacterial PDCs have so far only been found in mesophilic species. Other ASR studies described in the literature use a broader range of species with a wider growth-temperature range, including those by Hobbs et al. (2012) and Groussin et al. (2015).

It is possible that the mesophilic nature of ANC27 is caused by it simply being too young. On close examination of the study conducted by Gaucher et al. (2008) and their description of the progressive cooling of the environmental conditions correlating with a progressive decrease in the denaturing temperature of the bacterial elongation factor TU between 3.5 and 0.5 billion years ago, the denaturing temperature of $62^{\circ} \mathrm{C}$ for ANC27 fits well with their data. Their data suggest that in ancestors from 1000 to 2000 million years ago, a denaturing temperature of around $50^{\circ} \mathrm{C}$ is likely to be observed. However, earlier life (3-4 billion years ago) is likely to have experienced conditions similar to today's hot springs $\left(60-80^{\circ} \mathrm{C}\right.$; Gaucher et al., 2008). There are also reports of fluctuating trends in the thermophily of reconstructed enzymes, in which not all ancestors exhibit the exceptional thermal properties observed for other ancestors (Hobbs et al., 2012), and evidence for moderately thermophilic ancestors that show no specific trends with regard to catalytic activity or efficiency (Oulavallickal, 2016). ANC27 maybe more comparable with these enzymes.

In summary, we present the crystal structure of an inferred ancestral bacterial PDC, ANC27, and a brief functional and structural comparison with known extant bacterial PDCs. Structural analysis supports the previously observed correlation between decreased oligomeric interface and salt bridges and decreased thermostability. Although the ancestral protein ANC27 did not possess the thermostability that we hoped for, the suggestion that the lack of inter-dimer interactions appears to be the cause of its fragility should guide future ancestral reconstruction protocols to achieve the desired characteristics.

\section{Acknowledgements}

Special thanks to Konny Shim, University of Waikato, New Zealand for computational support.

\section{Funding information}

This work is based on a collaboration between the University of Bath, England and the University of Waikato, New Zealand supported by a Microbiology Society Research Visit Grant (RVG14-10). LB thanks the Biotechnology and Biological Sciences Research Council for a CASE studentship with TMO Renewables.

\section{References}

Abascal, F., Zardoya, R. \& Posada, D. (2005). Bioinformatics, 21, 2104-2105.

Adams, P. D. et al. (2010). Acta Cryst. D66, 213-221. 
Akanuma, S., Nakajima, Y., Yokobori, S.-I., Kimura, M., Nemoto, N., Mase, T., Miyazono, K.-I., Tanokura, M. \& Yamagishi, A. (2013). Proc. Natl Acad. Sci. USA, 110, 11067-11072.

Battistuzzi, F. U., Feijao, A. \& Hedges, S. B. (2004). BMC Evol. Biol. 4, 44.

Battye, T. G. G., Kontogiannis, L., Johnson, O., Powell, H. R. \& Leslie, A. G. W. (2011). Acta Cryst. D67, 271-281.

Bringer-Meyer, S., Schimz, K.-L. \& Sahm, H. (1986). Arch. Microbiol. 146, 105-110.

Buddrus, L., Andrews, E. S. V., Leak, D. J., Danson, M. J., Arcus, V. L. \& Crennell, S. J. (2016). Acta Cryst. F72, 700-706.

Castresana, J. (2000). Mol. Biol. Evol. 17, 540-552.

Chen, V. B., Arendall, W. B., Headd, J. J., Keedy, D. A., Immormino, R. M., Kapral, G. J., Murray, L. W., Richardson, J. S. \& Richardson, D. C. (2010). Acta Cryst. D66, 12-21.

Cole, M. F. \& Gaucher, E. A. (2011). Curr. Opin. Chem. Biol. 15, 399406.

Dobritzsch, D., König, S., Schneider, G. \& Lu, G. (1998). J. Biol. Chem. 273, 20196-20204.

Emsley, P., Lohkamp, B., Scott, W. G. \& Cowtan, K. (2010). Acta Cryst. D66, 486-501.

Evans, P. R. \& Murshudov, G. N. (2013). Acta Cryst. D69, 1204-1214.

Gaucher, E. A., Govindarajan, S. \& Ganesh, O. K. (2008). Nature (London), 451, 704-707.

Gocke, D., Graf, T., Brosi, H., Frindi-Wosch, I., Walter, L., Müller, M. \& Pohl, M. (2009). J. Mol. Catal. B Enzym. 61, 30-35.

Groussin, M., Hobbs, J. K., Szöllősi, G. J., Gribaldo, S., Arcus, V. L. \& Gouy, M. (2015). Mol. Biol. Evol. 32, 13-22.

Guindon, S., Dufayard, J.-F., Lefort, V., Anisimova, M., Hordijk, W. \& Gascuel, O. (2010). Syst. Biol. 59, 307-321.

Hobbs, J. K., Prentice, E. J., Groussin, M. \& Arcus, V. L. (2015). J. Mol. Evol. 81, 110-120.

Hobbs, J. K., Shepherd, C., Saul, D. J., Demetras, N. J., Haaning, S., Monk, C. R., Daniel, R. M. \& Arcus, V. L. (2012). Mol. Biol. Evol. 29, 825-835.

Huynh, K. \& Partch, C. L. (2015). Curr. Protoc. Protein Sci. 79, 28.9.1-28.914.

Kearse, M., Moir, R., Wilson, A., Stones-Havas, S., Cheung, M., Sturrock, S., Buxton, S., Cooper, A., Markowitz, S., Duran, C., Thierer, T., Ashton, B., Meintjes, P. \& Drummond, A. (2012). Bioinformatics, 28, 1647-1649.

Krissinel, E. \& Henrick, K. (2007). J. Mol. Biol. 372, 774-797.
Long, F., Vagin, A. A., Young, P. \& Murshudov, G. N. (2008). Acta Cryst. D64, 125-132.

Lowe, S. E. \& Zeikus, J. G. (1992). J. Gen. Microbiol. 138, 803-807.

Löytynoja, A. \& Goldman, N. (2010). BMC Bioinformatics, 11, 579.

Merkl, R. \& Sterner, R. (2016). Biol. Chem. 397, 1-21.

Meyer, D., Neumann, P., Parthier, C., Friedemann, R., Nemeria, N., Jordan, F. \& Tittmann, K. (2010). Biochemistry, 49, 81978212.

Murshudov, G. N., Skubák, P., Lebedev, A. A., Pannu, N. S., Steiner, R. A., Nicholls, R. A., Winn, M. D., Long, F. \& Vagin, A. A. (2011). Acta Cryst. D67, 355-367.

Oulavallickal, T. (2016). PhD Thesis. University of Waikato, Hamilton, New Zealand.

Pauling, L. \& Zuckerkandl, E. (1963). Acta Chem. Scand. 17, S9S16.

Pei, J., Erixon, K. M., Luisi, B. F. \& Leeper, F. J. (2010). Biochemistry, 49, 1727-1736.

Perez-Jimenez, R., Inglés-Prieto, A., Zhao, Z.-M., Sanchez-Romero, I., Alegre-Cebollada, J., Kosuri, P., Garcia-Manyes, S., Kappock, T. J., Tanokura, M. \& Holmgren, A. (2011). Nature Struct. Mol. Biol. 18, 592-596.

Posada, D. (2008). Mol. Biol. Evol. 25, 1253-1256.

Potterton, L. et al. (2018). Acta Cryst. D74, 68-84.

Raj, K. C., Talarico, L. A., Ingram, L. O. \& Maupin-Furlow, J. A. (2002). Appl. Environ. Microbiol. 68, 2869-2876.

Risso, V. A., Gavira, J. A., Mejia-Carmona, D. F., Gaucher, E. A. \& Sanchez-Ruiz, J. M. (2013). J. Am. Chem. Soc. 135, 2899-2902.

Sanderson, M. J. (2003). Bioinformatics, 19, 301-302.

Siegert, P., McLeish, M. J., Baumann, M., Iding, H., Kneen, M. M., Kenyon, G. L. \& Pohl, M. (2005). Protein Eng. Des. Sol. 18, 345357.

Wechsler, C., Meyer, D., Loschonsky, S., Funk, L. M., Neumann, P., Ficner, R., Brodhun, F., Muller, M. \& Tittman, K. (2015). Chembiochem, 16, 2580-2584.

Winn, M. D. et al. (2011). Acta Cryst. D67, 235-242.

Yang, Z. (2007). Mol. Biol. Evol. 24, 1586-1591.

Zwickl, D. J. (2010). PhD Thesis. The University of Texas, Austin, Texas, USA.

Zyl, L. J. van, Schubert, W. D., Tuffin, M. I. \& Cowan, D. A. (2014). BMC Struct. Biol. 14, 21.

Zyl, L. J. van, Taylor, M. P., Eley, K., Tuffin, M. \& Cowan, D. A. (2014). Appl. Microbiol. Biotechnol. 98, 1247-1259. 